\title{
Interference Coordination between Femtocells in LTE-Advanced Networks with Carrier Aggregation
}

\author{
Kan Zheng ${ }^{\dagger}$, Fanglong $\mathrm{Hu}^{\dagger}$, Lei $\mathrm{Lei}^{\ddagger}$, and Wenbo Wang ${ }^{\dagger}$ \\ ${ }^{\dagger}$ Wireless Signal Processing and Network Lab \\ Key laboratory of Universal Wireless Communication, Ministry of Education \\ Beijing University of Posts\&Telecommunications \\ Beijing, China, 100088 \\ $\ddagger$ Wireless System Lab \\ China Mobile Research Institute \\ Beijing, China, 100053
}

\begin{abstract}
Femtocells technology has emerged as a promising solution to provide the indoor coverage in the 3rd generation (3G) long-term evolution (LTE) and its advanced (LTE-Advanced) networks. In this paper, we propose a two-step interference coordination scheme to deal with the interference between femtocells in LTE-Advanced networks with carrier aggregation. Each femtocell is assigned a carrier that is non-overlapping with its interfering femtocells or has the least interference in the first step. Then, in the second step, more carriers are attempted to be used by a femtocell in order to improve the system spectrum efficiency based on the specified utility functions. Our simulation results demonstrate the effectiveness of the proposed interference coordination scheme in terms of throughput.
\end{abstract}

Index Terms-Femtocell; Interference Coordination; Carrier Aggregation.

\section{INTRODUCTION}

As a cost-efficient indoor coverage solutions, femtocell technology has been proposed and applied in the 3rd Generation Partnership Project (3GPP) for its Universal Mobile Telecommunications System (UMTS), long-term evolution (LTE) networks and its advancement (LTE-Advanced) [1], [2]. Not only users but also operators can benefit from the femtocell solutions. Compared with macrocells, the femtocells provide better radio link quality to the users while consuming less power of mobile devices in indoor environments. For operators, a significant amount of traffic can be moved from the macrocell network to the femtocell network, thus reducing the deployment cost. However, this technology is still under development and there are numerous problems needed to be investigated such as access control, timing and synchronization, and interference coordination [3].

Since femtocell networks are customer-deployed without proper network planning, their interference environment tends to be much more complicated than traditional cellular networks. Thus, the interference coordination in femtocell networks cannot be handled by the existing schemes designed for macrocell deployments. Generally, there exist two kinds of interferences in orthogonal frequency division multiplexing access (OFDMA) femtocell networks, i.e. interference between the macrocell and femtocell and the femtocell-to- femtocell interference. Orthogonalization of resources in frequency domain and/or in time domain for the macrocell and femtocell can avoid the interference between macrocell and femtocell although it is inefficient in term of spectrum reuse [4]. So, in [5], a frequency assignment scheme was proposed for femtocell networks while considering the handoff and interference between the macrocell and femtocell. On the other hand, since the number and position of the base stations in femtocell networks are semi-statically varying, the femtocell-to-femtocell co-channel interference has to be dealt with by specifically designed radio resource allocation scheme. However, to the best of our knowledge, the resource allocation problem for coordinating the interference between femtocells has not been explicitly addressed in prior literature so far.

Meanwhile, carrier aggregation, where two or more component carriers are aggregated, has been already adopted by LTEAdvanced in order to support wider transmission bandwidths and for spectrum aggregation [2]. In this paper, we first formulate the interference coordination between femtocells in LTE-Advanced as an optimization problem, which is quite hard to be solved directly. Instead, a CA-based interference coordination scheme is proposed to deal with the interference between femtocells in LTE-Advanced networks, where the femtocell base station and user is usually refer to home evolved NodeB (HeNB) and home user equipment (HUE), respectively. Based on the measurement at HeNB, each HeNB is assigned to a carrier that is non-overlapping with its interfering HeNBs or has the least interference in the first step. Then, in order to increase the spectrum efficiency of the network, the carriers already used by a HeNB is possibly shared to other HeNBs based on the utility function calculated at HUE in the second step. Finally, our simulation results demonstrate the effectiveness of the proposed scheme in terms of throughput.

This paper is organized as follows. Section II gives a brief overview of the system and problem formulation. The CAbased interference coordination scheme is proposed in Section III. Then, Section IV gives the simulation results. Finally, Section V concludes this paper. 


\section{SySTEM MODEL}

The downlink LTE-Advanced network with femtocells is considered in this paper. There are usually few users with very low or even no mobility in the home or office environment, so that only one HUE is assumed to be connected to the HeNB per cell for simplicity [6]. The analytical method can also be easily extended to the cases with more than one UE per cell.

Consider the downlink transmission with $N$ users and $K$ carriers in the network. For simplicity, the transmit power is kept the same in each carrier per cell, i.e. $P_{T}$. Let the binary matrix $\mathbf{A}=\left\{a_{k, n} \mid a_{k, n} \in\{0,1\}\right\}_{K \times N}$ describe the resource allocation among the users, where $a_{k, n}=1$ denotes that carrier $k$ is assigned to user $n$, otherwise $a_{k, n}=0$. Then, the achievable rate on carrier $k$ in femtocell $n$ is given by

$$
\begin{array}{r}
\eta_{k, n}=W \log _{2}\left(1+\frac{L_{n, n} P_{T}}{\sum_{j=1, j \neq n}^{N} L_{n, j} a_{k, j} P_{T}+\sigma_{N}^{2}}\right), \\
1 \leq k \leq K, 1 \leq n \leq N,
\end{array}
$$

where $W$ is the carrier bandwidth, $L_{n, j}$ is the pathloss (PL) from the $j$ th user's serving HeNB to UE $n$, and $\sigma_{N}^{2}$ is the noise power of the additive white Gaussian noise (AWGN).

The interference coordination problem is to find $\mathbf{A}$ such that an objective function is optimized. Usually, the following optimization problems with the different objectives are needed to be solved for interference coordination, i.e.

- Maximize Throughput (Max-TP). Assuming the goal is to achieve the highest possible system spectrum efficiency, the objective function of this problem can be formulated as

$$
\max _{\mathbf{A}} \sum_{n=1}^{N} \sum_{k=1}^{K} a_{k, n} \eta_{k, n} .
$$

- Maximize Proportional-Fair (Max-PF). Assuming the goal is to achieve the highest system throughput while ensuring proportional fairness among femtocells, the sum of the logarithmic average cell throughput needs to be maximized [7], i.e.

$$
\max _{\mathbf{A}} \sum_{n=1}^{N} \log \left(\sum_{k=1}^{K} a_{k, n} \cdot \eta_{k, n}\right) .
$$

By integrating (1) into (2) or (3), we can easily find that the optimization problem is non-concave. To find its optimal solution, exhaustive search over all the possible solution set is needed, which has prohibitively high computational complexity.

Therefore, considering the implementation feasibility, it is necessary to deal with the interference coordination problem in the LTE-Advanced network by other methods.

\section{INTERFERENCE COORDINATION BETWEEN FEMTOCELLS}

Since the behaviors of HeNBs in femtocell networks are controlled by the customers, the network topology of femtocell systems varies semi-statically, resulting in complex femtocell-to-femtocell co-channel interferences. The interferences among femtocells have to be coordinated in order to achieve a good balance between an aggressive reuse of the spectral resource and the resulting co-channel interferences. A CA-based two-step interference coordination scheme is proposed in this section. To ensure the fairness between femtocells, only one carrier is assigned to each femtocells in the first step based on the measurement of the inter-cell interference. Then, more carriers are attempted to be used by a femtocell in order to improve the system spectrum efficiency in the second step.

\section{A. Step 1: Orthogonal frequency partitioning (OFP)}

When a HeNB is powered on, it measures the Reference Signal Received Power (RSRP) of the surrounding HeNBs and determines the set of HeNBs that are likely to have an interfering relationship with it by checking whether the RSRP is above a certain threshold, i.e. $\Gamma_{O F P}$. Then, the HeNB will be assigned a carrier that is non-overlapping with its interfering HeNBs. If all the carriers are already occupied by its interfering HeNBs, the HeNB shall select the carrier with the least interference, e.g. the carrier on which the sum of RSRP from the surrounding HeNBs is the smallest. The carrier that is assigned to the HeNB in this step is referred to as its orthogonal carrier. After this step, one carrier has been allocated to each femtocell, which provides the communication connection between the HeNB and UE.

\section{B. Step 2: Adaptive frequency reuse (AFR)}

Based on the measurement results of the HUEs, HeNB $n$ decides whether it can share the carriers used by its interfering HeNBs in this step. Since we assume that there is only one HUE associated with a HeNB, the HUE and HeNB can share the same index for notation simplicity. HUE $n$ measures not only the RSRP of its serving HeNB but also the RSRPs of its interfering HeNBs. Then, if its serving HeNB, i.e. HeNB $n$, uses an orthogonal carrier $k$ of its interfering HeNBs, the signal-interference-ratio (SIR) of HUE $n$ on carrier $k$ can be calculated as:

$$
\xi_{n}^{(k)}=\frac{r_{n, n}}{\sum_{m \in \mathcal{I}_{n}^{(k)}} r_{n, m}}
$$

where $r_{n, n}$ and $r_{n, m}$ denote the RSRP measured at HUE $n$ from HeNB $n$ and $m$, respectively, and $\mathcal{I}_{n}^{(k)}$ represents the set of interfering HeNBs of HeNB $n$ which currently uses carrier $k$. If its SIR is above a certain threshold (e.g. the demodulation threshold of the data channel), HeNB $n$ decides it can use the carrier $k$. It then sends a frequency reuse request to those HeNBs in $\mathcal{I}_{n}^{(k)}$ which are currently using carrier $k$. The frequency reuse request message carries a utility value, which is determined by the different objectives. Corresponding to either Max-TP or Max-PF objective, the utility can be calculated by either of the following two formulas as

- Type A:

$$
U_{k, n}^{A}=\frac{g_{n}^{(k)}}{\left\|\mathcal{I}_{n}^{(k)}\right\|},
$$




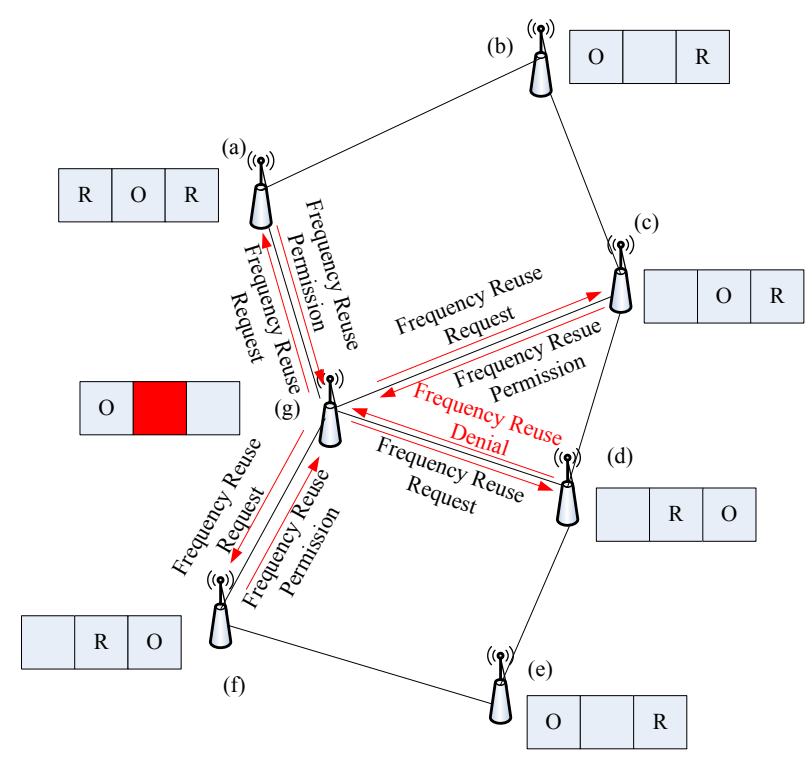

Fig. 1. An example of the AFR procedure.

- Type B:

$$
U_{k, n}^{B}=\frac{g_{n}^{(k)}}{\tilde{\eta}_{n} \cdot\left\|\mathcal{I}_{n}^{(k)}\right\|},
$$

where $g_{n}^{(k)}$ is the throughput gain of HUE $n$ if carrier $k$ is granted to it (i.e. the throughput of HUE $n$ on carrier $k$ which is the function of $\left.\xi_{n}^{(k)}\right), \tilde{\eta}_{n}$ is the current throughput of HeNB $n$ without using carrier $k$, and $\left\|\mathcal{I}_{n}^{(k)}\right\|$ represents the size of set $\mathcal{I}_{n}^{(k)}$. While (5) only considers maximizing the sum throughput of all the femtocells, (6) also considers the fairness issue.

When a HeNB $m$ receives the frequency reuse request, it can calculate the SIR of its associated UE $m$ on carrier $k$, i.e. $\xi_{m}^{(k)}$, while assuming that this carrier is granted to HeNB $n$ Then, HeNB $m$ calculates a utility value with either of the following two formulas as

- Type A:

$$
\tilde{U}_{k, m}^{A}=g_{m}^{(k)},
$$

- Type B:

$$
\tilde{U}_{k, m}^{B}=\frac{g_{m}^{(k)}}{\tilde{\eta}_{m}} .
$$

Note that compared with (5) and (6), the size of interfering HeNBs set does not exist in the denominator. This is because if carrier $k$ is granted to $\operatorname{HeNB} n$, all the $\left\|\mathcal{I}_{n}^{(k)}\right\|$ interfering HeNBs will have throughput degradation while only HeNB $n$ has throughput gain.

If the utility of HeNB $m$ is less than that of HeNB $n$, HeNB $m$ decides that carrier $k$ can be granted to HeNB $n$. It then sends a frequency reuse permission to HeNB $n$. Otherwise, it sends a frequency reuse denial to HeNB $n$. If HeNB $n$ receives the frequency reuse permissions from all the HeNBs in $\mathcal{I}_{n}^{(k)}$, it can start to use carrier $k$, which is referred to as its reuse carriers. Otherwise, it does not use this carrier.

Fig.1 illustrates an example of the AFR process, where three carriers are assumed to exist in the network. For notation,

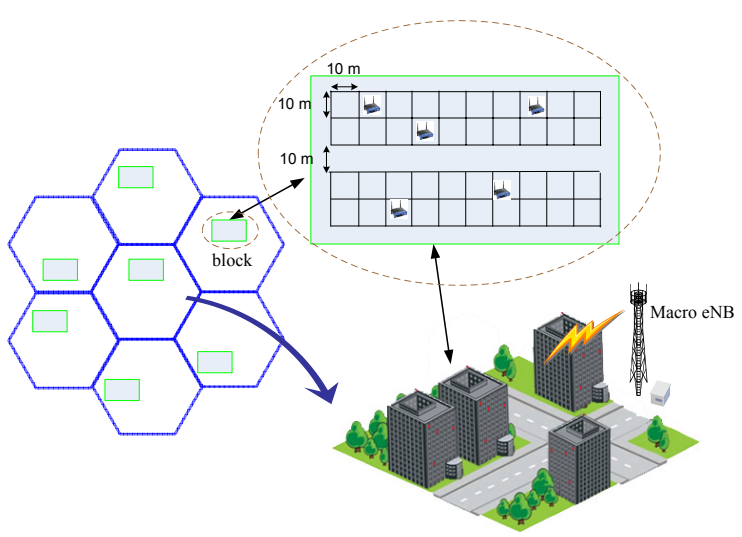

Fig. 2. Femtocells deployment in LTE-Advanced networks.

TABLE I

PARAMETERS ASSUMPTION IN LTE-ADVANCED WIRELESS NETWORKS WITH FEMTOCELLS.

\begin{tabular}{|l|l|}
\hline Parameters & Values \\
\hline Carrier bandwidth & $5 \mathrm{MHz}$ \\
\hline Number of Carriers & 3 \\
\hline Number of Subchannel & 50 \\
\hline Min separation UE to HeNB & $0.2 \mathrm{~m}$ \\
\hline Number transmit antennas HeNB & 1 \\
\hline Number receive antennas UE & 2 \\
\hline HeNB antenna gain & $5 \mathrm{dBi}$ \\
\hline Path loss model HeNB to UE (dB) & Complex Model 2 [2] \\
\hline Shadowing standard deviation & LOS:3 dB/NLOS $4 \mathrm{~dB}$ \\
\hline Noise figure at UE & $9 \mathrm{~dB}$ \\
\hline Transmit power HeNB & $20 \mathrm{dBm}$ \\
\hline Number of user per femtocell & 1 \\
\hline Traffic model & Full Buffer \\
\hline
\end{tabular}

"O" stands for orthogonal carriers while " $\mathbf{R}$ " stands for the reuse carrier. After HeNB $g$ is powered on and has selected its orthogonal carrier, it examines whether it can reuse the rest of the carriers with its neighbors. In this example, we assume that the second carrier is the candidate reuse carrier of HeNB $g$. Then, HeNB $g$ send a "Frequency Reuse Request" signalling to other HeNBs belonging to the set $\mathcal{I}_{g}^{(2)}$, which are using the second carrier. In the request signalling message, HeNB $g$ sends the value of its $U_{2, g}^{A}$ or $U_{2, g}^{B}$. After receiving the request signalling, the HeNBs in the set $\mathcal{I}_{g}^{(2)}$, i.e., HeNB $a, c, d$ and $f$, calculate their $\tilde{U}_{2, m}^{A}$, or $\tilde{U}_{2, m}^{B},(m=a, c, d, f)$ values. When $\tilde{U}_{2, m}^{A}<U_{2, g}^{A}$, or $\tilde{U}_{2, m}^{B}<U_{2, g}^{B}$, HeNB $m$ will send a "Frequency Reuse Permission" signalling to HeNB $g$; otherwise, it will send a "Frequency Reuse Denial" signalling. HeNB $g$ cannot use the second carrier until all the HeNBs feedback "Frequency Reuse Permission" signalling messages.

\section{Simulation Results}

In this section, simulation results are presented to evaluate the performances of the proposed interference coordination scheme in LTE-Advanced networks with femtocells. For comparison purpose, the performances of the network 


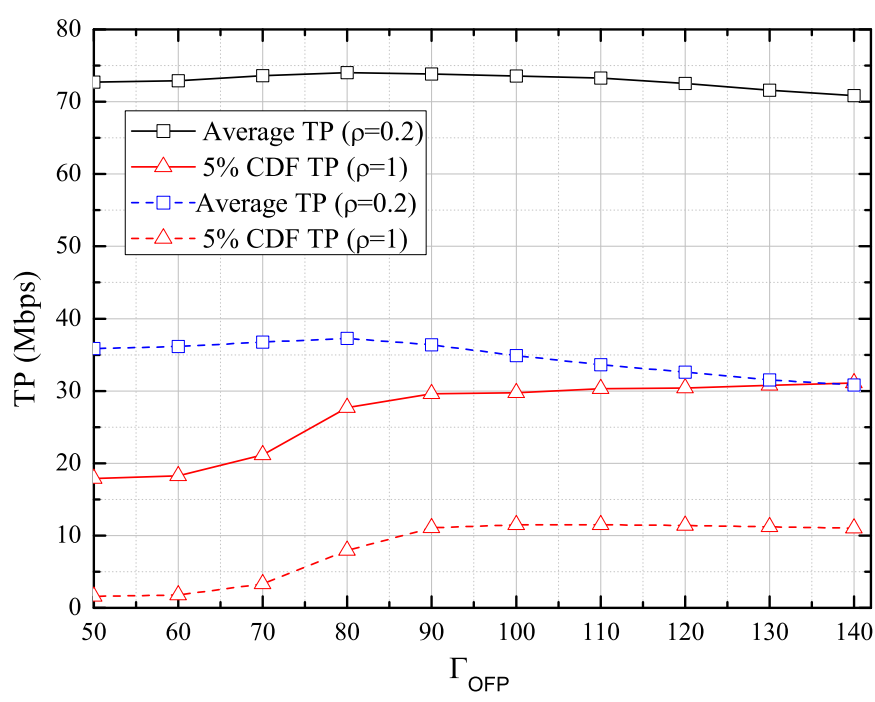

Fig. 3. Throughput performances with different threshold in OFP.

with all the carriers fully reused between HeNBs are also given. We consider the dense-urban scenario corresponding to densely-populated areas, where there are multi-floor apartment buildings with small size apartment units as shown in Fig.2. Detailed simulation parameters including channel model and system assumptions are summarized in Table I [2]. For simplicity, only one femtocell block is considered. As shown in Fig.2, each femtocell block consists of two stripes of apartments, and each stripe has 2 by $N_{D}$ apartments (e.g. $N_{D}$ is set to 10 as illustrated in Fig.2). Each apartment with the size of $10 m \times 10 m$ may have a femtocell. There is a street with width of $10 \mathrm{~m}$ between two stripes of apartments. Each femtocell block has $L_{D}$ floors, which can be chosen randomly between 1 and 10 (e.g. $L_{D}=6$ in our simulations).

To simulate the realistic case where an apartment may not have a femtocell, a parameter of deployment ratio $\rho$ is used to determine whether an apartment is deployed with a femtocell or not. Since femtocells are not always on, we defined another parameter of activation ratio $\beta$ to describe the percentage of active femtocells. Only when a femtocell is active, it will transmit with the given power at traffic subchannels. Otherwise, it will keep silent at traffic subchannels. The activation ratio $\beta$ can be varied from 0 to $100 \%$. Both the HeNB and the UE are dropped uniformly at random in the apartment. In our simulations, the activation ratio $\beta$ is assumed to be $50 \%$ while the deployment ratio $\rho$ is 0.2 or 1 .

In Fig.3, we present the average throughput (TP) performances of the network with our proposed channel allocation method while using different values of threshold in the first step, i.e. OFP. With the smaller value of the threshold, more average throughput can be achieved because carriers are reused by femtocells more frequently. Such throughput varying is little when the threshold is smaller than $90 \mathrm{~dB}$. On the other hand, the femtocells with low SINRs are more sensible to the interference so that their throughput performances become smaller with the decrease of the threshold even when more carriers are allocated. So, the throughput performance at the

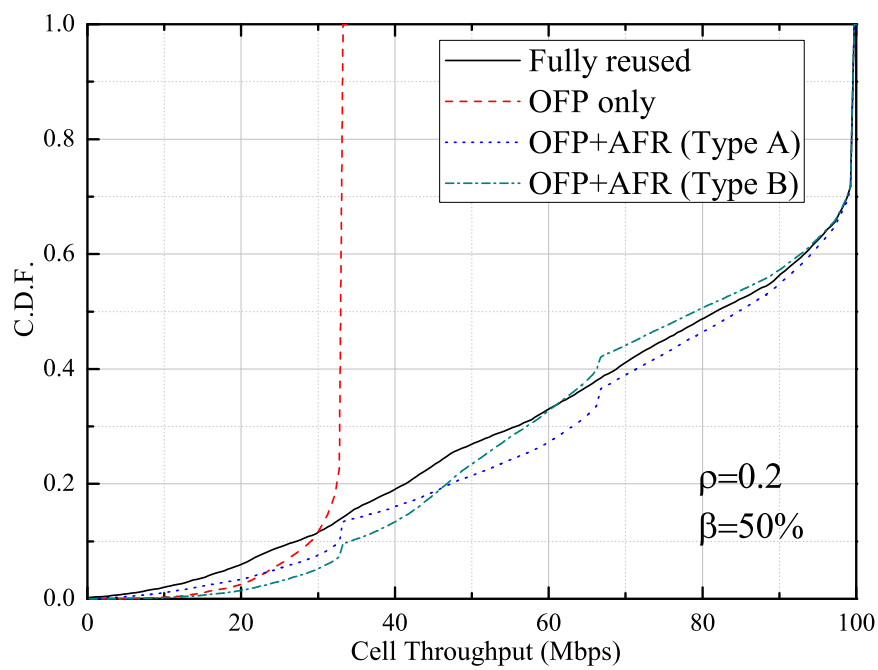

Fig. 4. CDF of cell throughput with/without the proposed interference coordination scheme in the network with $\rho=0.2$ and $\beta=50 \%$.

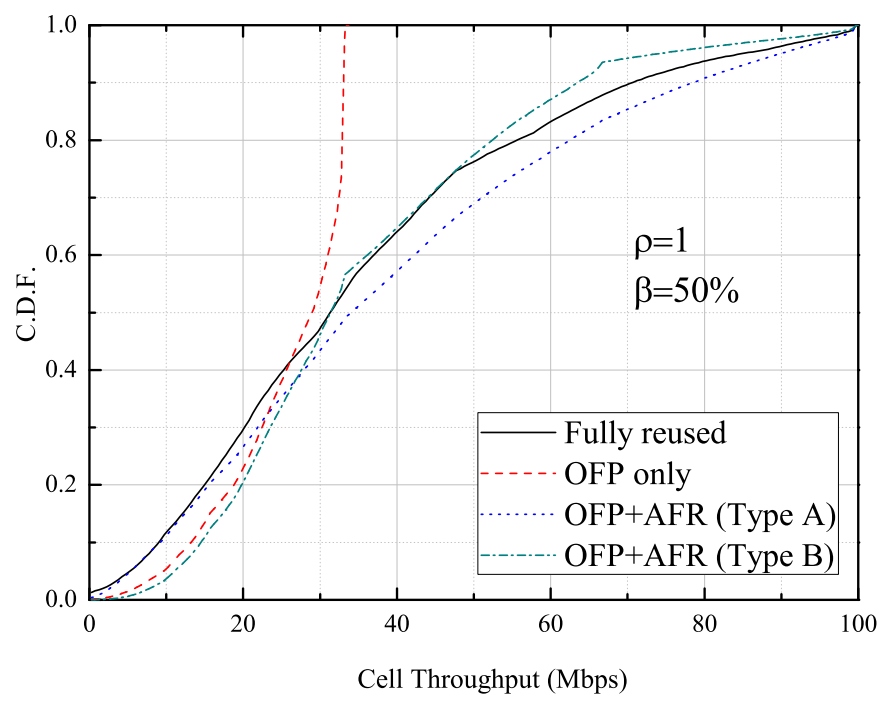

Fig. 5. CDF of cell throughput with/without the proposed interference coordination scheme in the network with $\rho=1$ and $\beta=50 \%$.

5\% Cumulative distribution function (CDF) becomes a little worse when the threshold is less than $90 \mathrm{~dB}$. Therefore, we select the threshold value as $90 \mathrm{~dB}$ in the following simulations.

Next, the CDF performances of the networks with or without the proposed interference coordination scheme are compared in Fig.4 and Fig.5, where the deployment ratio $\rho$ is 0.2 and 1 , respectively. Compared to the networks where carriers are fully reused between femtocells, the throughput performance of the networks with the proposed scheme is better no matter which utility function is applied. Moreover, in order to clearly verify the effects of different steps in the proposed scheme, we also present the performance of the network with only the first step, i.e. OFP. Since only one carrier is allocated by OPF to each femtocell, the corresponding poor throughput is expected as shown in Fig.4 and Fig.5. The 
TABLE II

THROUGHPUT COMPARISON BETWEEN DIFFERENT SCHEMES IN LTE-ADVANCED NETWORKS WITH FEMTOCELLS.

\begin{tabular}{|c|c|c|c|c|}
\hline & \multicolumn{2}{|c|}{ Average TP (Mbps) } & \multicolumn{2}{c|}{ 5\% CDF TP (Mbps) } \\
\cline { 2 - 5 } & $\rho=0.2$ & $\rho=1$ & $\rho=0.2$ & $\rho=1$ \\
\hline Fully reused & 72.2420 & 35.9421 & 18.0160 & 5.2953 \\
\hline OFP+AFR (Type A) & 75.0090 & 39.5478 & 24.3308 & 5.3974 \\
\hline OFP+AFR (Type B) & 74.0236 & 36.3689 & 30.1927 & 11.0766 \\
\hline
\end{tabular}

purpose of the OFP is to ensure that each femtocell has at least one carrier to be used.

The average cell throughput and the 5\% CDF throughput in the networks with or without the proposed scheme are also compared in Table II. The performances of the network with fully reused scheme are affected by the interference between femtocells especially for the low SINR HUEs. We can observe that gains due to the proposed scheme exist on not only the average cell throughput but also the 5\% CDF throughput. From viewpoint of average throughput, the proposed scheme with Type A utility can achieve the best performance. However, it does not consider the fairness between femtocells. We can find that not only the average throughput but also the 5\% CDF throughput in the network using the proposed scheme with Type $B$ utility are much better than that with fully reused method.

\section{CONCLUSION}

In this paper, we first present the interference coordination problem in LTE-Advanced networks with femtocells. Then, a practical CA-based scheme with two steps is proposed to deal with the interference between femtocells. To ensure the fairness between femtocells, only one carrier is assigned to each femtocells based on the measurement of the inter-cell interference in the first step . Then, after calculating either of two proposed utility functions, more carriers are attempted to be used by a femtocell in order to improve the system spectrum efficiency in the second step. Our simulation results show that the proposed interference coordination scheme outperforms the fully reused scheme. Especially, not only the average throughput but also the $5 \% \mathrm{CDF}$ throughput can be quite improved by the proposed scheme with Type $B$ utility. This scheme can be easily extended to the cases with the smaller radio resource unit instead of carrier.

\section{ACKNOWLEDGMENT}

This work was supported in part by China Mobile Research Institute and National Key Technology R\&D Program of China under Grant 2009ZX03003-008-01.

\section{REFERENCES}

[1] D. N. Knisely, T. Yoshizawa, and F. Favichia, "Standardization of femtocells in 3GPP," IEEE Communications Magazine, vol. 47, no. 9, pp. $68-75$, Sept. 2009.

[2] 3GPP TR 36.814, v2.0.0, "Further Advancements for E-UTRA, Physical Layer Aspects", Mar. 2010.

[3] V. Chandrasekhar, J. Andrews, and A. Gatherer, "Femtocell networks: a survey" IEEE Communications Magazine, vol. 46, no. 9, pp. 59-67, Sept. 2008.
[4] V. Chandrasekhar and J. G. Andrews, "Spectrum allocation in twotier networks," Asilomar Conference on Signals, Systems and Computers, 2008, pp. 1583-1587, Oct. 2008.

[5] I. Guvenc, J. Moo-Ryong, F. Watanabe, and H. Inamura, "A hybrid frequency assignment for femtocells and coverage area analysis for cochannel operation," IEEE Communications Letters, vol. 12, no. 12, pp. 880-882, Dec. 2008

[6] Femto Forum Working Group 2 Document, "OFDMA Interference Study: Evaluation Methodology Document," Mar. 2009.

[7] Jean-Yves Boudec, "Rate adaptation, Congestion Control and Fairness: A Tutorial," http://www.epfl.ch/PS_files/LEB3132.pdf. 